\title{
Trasplante pulmonar en pacientes con fibrosis pulmonar. Experiencia del Instituto Nacional del Tórax
}

\author{
CLAUDIA SEPÚLVEDA L.*, VÍCTOR LEIVA V.*, VIRGINIA LINACRE S.*, MAURICIO SALINAS F.*, \\ PEDRO UNDURRAGA M.*, JAQUELINE LÓPEZ R.*, CATALINA BRICEÑO V.**, \\ FRANCESCA GAJARDO Z.*, ERIKA DONOSO G.* y JOEL MELO T.*
}

\section{Lung transplantation in pulmonary fibrosis. An experience from National Institute of Thorax, Chile}

Pulmonary fibrosis is a progressive disease with a bad prognosis. This situation makes rise lung transplant as a therapeutic option among carefully selected patients. Objective: Evaluate the results and survival rates of patients with pulmonary fibrosis that were transplanted through an 8 years period of follow-up, from the beginning of our transplant program. Methods: Descriptive study of the transplanted patients diagnosed with pulmonary fibrosis from august 2010 to july 2018. Results: Out of 76 transplants, $68.4 \%$ were due to pulmonary fibrosis, among these, the main diagnosis was idiopathic pulmonary fibrosis (75\%). The average lung allocation score (LAS) was 53 and $32 \%$ of them had urgency criteria. Patients' age averaged 55 years-old and $98 \%$ of them underwent a single lung transplant. Early medical complications were seen in 26 patients with infectious episodes and 6 with acute rejection. The main late complication was chronic allograft dysfunction. The main surgical complication was bronchial stenosis (7.6\%). In comparison to its base line reference values FVC means pre transplant and 1 and 3 years post-transplant were 49\%, $70 \%$ and $71 \%$ respectively. A $40 \%$ of patients died during follow up period. Infections were the main cause of mortality during the first year. Survival rates at $1^{\text {st }} 3^{\text {rd }}$ and $5^{\text {th }}$ year were $86,2 \%$; 65.2\% and 59.8\% respectively. Conclusions: Single lung transplant is a therapeutic option for patients with interstitial lung disease with a 59\% survival rate in 5 years, 1/3 fulfilled urgency criteria at the transplant time. The infections were the main early complication and chronic graft dysfunction was the main late complication.

Key words: Lung transplantation; idiopathic pulmonary fibrosis; survival rate; follow-up studies.

\section{Resumen}

La fibrosis pulmonar es una enfermedad progresiva y de mal pronóstico por lo que el trasplante pulmonar sigue siendo una opción para pacientes bien seleccionados. Objetivo: Evaluar resultados y sobrevida de pacientes con fibrosis pulmonar trasplantados a 8 años de inicio del programa de trasplante. Métodos: Estudio descriptivo de trasplantados de pulmón por fibrosis pulmonar desde agosto de 2010 a julio de 2018. Resultados: De un total de 76 trasplantes, un 68,4\% han sido en pacientes con fibrosis pulmonar. La principal indicación fue fibrosis pulmonar idiopática (75\%). El puntaje de priorización pulmonar (LAS) promedio fue de 53 y un 32\% cumplía con criterios de urgencia. La edad promedio fue 55 años, y se usó técnica unipulmonar en un 98\%. La principal complicación quirúrgica fue la estenosis bronquial (7,6\%). De las complicaciones médicas precoces destacaron 26 episodios infecciosos y 6 rechazos celulares agudos. La principal complicación tardía fue la disfunción crónica de injerto. Los resultados funcionales promedio pre trasplante, a 1 y 3 años fueron: CVF de 49\%, 71\% y $70 \%$ del valor teórico. Un $40 \%$ ha fallecido en el período de seguimiento. La principal causa de

\footnotetext{
* Instituto Nacional del Tórax. Santiago,Chile.

** Departamento Enfermedades Respiratorias. Pontificia Universidad Católica de Chile. Santiago,Chile.
} 
mortalidad el primer año post trasplante fueron las infecciones. La sobrevida a 1, 3 y 5 años fue de 86,2\%, 65,2\% y 59,8\% respectivamente. Conclusiones: En trasplante monopulmonar es una opción de tratamiento en estos pacientes, con una sobrevida de 59\% a 5 años. Un tercio se trasplanta con criterios de urgencia, siendo las infecciones la principal complicación precoz y la disfunción crónica de injerto la complicación tardía más frecuente.

Palabras clave: Trasplante pulmonar, fibrosis pulmonar idiopática; tasa de supervivencia; estudios de seguimiento.

\section{Introducción}

La Fibrosis Pulmonar Idiopática (FPI) es una enfermedad progresiva y de mal pronóstico cuya sobrevida promedio es de 2 a 5 años tras el diagnóstico. A pesar de que en los últimos años han sido aprobadas drogas que demuestran enlentecer la progresión de la enfermedad, el trasplante pulmonar (TP) sigue siendo la mejor opción terapéutica en pacientes con fibrosis pulmonar (FP) con enfermedad avanzada. Siendo recomendada en las actuales guías de la Sociedad Internacional de Trasplante de Corazón y Pulmón (ISHLT) una derivación temprana de estos pacientes a un centro de trasplante ${ }^{1}$.

Debido a una mayor mortalidad de pacientes con FPI en lista de espera, en el año 2005 se implementó en Estados Unidos, un nuevo sistema de puntaje en la asignación de órganos (LAS: Lung Allocation Score) que dado los parámetros que utiliza para su cálculo, permite priorizar a pacientes con fibrosis pulmonar que tienen alto riesgo de morir dentro del año, lo que ha favorecido que un número creciente de pacientes con esta enfermedad se trasplanten.

Según el registro de la ISHLT la principal indicación actual de trasplante pulmonar son las enfermedades pulmonares difusas $(31,3 \%)$, siendo el trasplante bipulmonar el que ha demostrado mejores resultados en los análisis de los últimos años en términos de sobrevida a largo plazo ${ }^{2}$.

En Chile, el Instituto Nacional del Tórax (INT) es el único centro público que realiza trasplante pulmonar desde el año 2010 y el objetivo de este estudio es evaluar los resultados y sobrevida de pacientes con FP a 8 años de inicio del programa.

\section{Métodos}

Estudio descriptivo con revisión de registros clínicos de pacientes trasplantados de pulmón por FP desde agosto de 2010 a julio de 2018 en el Instituto Nacional del Tórax. Se consignaron las características demográficas, tipo de fibrosis pulmonar, presencia de hipertensión pulmonar, puntaje LAS, tipo de trasplante, uso de soporte extracorpóreo intraoperatorio, complicaciones quirúrgicas y médicas precoces ( $<6$ meses) y tardías, función pulmonar basal y post trasplante, sobrevida a 1, 3 y 5 años y causas de muerte.

El diagnóstico pre trasplante de FPI, alveolitis alérgica extrínseca (AAE) y neumonía intersticial con características de autoinmunidad (IPAF) son realizados por un equipo multidisciplinario de enfermedades intersticiales del INT basados en las guías de la ATS/ERS/ALAT y confirmadas histológicamente con las biopsias del explante . $^{3}$

Desde el año 2017 se utiliza el puntaje de asignación de órganos LAS a nivel nacional como forma de priorizar a pacientes en lista de espera, previo a esa fecha la opción de trasplante se basaba en tiempo de ingreso en lista y grupo sanguíneo. Este puntaje se calculó retrospectivamente en pacientes TP previo a enero de 2017. Un puntaje $>45$ fue considerado como criterio de urgencia dado que refleja a pacientes con enfermedad avanzada y mayor riesgo de muerte ${ }^{4}$.

Se definió hipertensión arterial pulmonar por criterios ecocardiográficos como la presencia de una PSAP (presión sistólica arterial pulmonar) $>40 \mathrm{mmHg}$.

Para el diagnóstico de disfunción crónica del aloinjerto (CLAD) y sus dos fenotipos síndrome de bronquiolitis obliterante (BOS) y síndrome del injerto restrictivo (RAS) nos guiamos por las recomendaciones internacionales ${ }^{5}$.

La indicación de trasplante se basó en el consenso de selección de candidatos a trasplante de pulmón de la ISHLT ${ }^{1}$.

En nuestro programa realizamos fibrobroncoscopía flexible (FBC) de seguimiento, con toma de cultivos y biopsias transbronquiales (BTB) en los meses 1-3-6-12 y 18 y en casos de duda diagnóstica entre infección o rechazo. El diagnóstico de rechazo celular y/o humoral se realizó mediante FBC con BTB, análisis histopatológico de la muestra, tinción $\mathrm{C} 4 \mathrm{~d}$ en biopsia y presencia de anticuerpos donante específicos, siguiendo las recomendaciones internacionales ${ }^{6}$. Los casos de rechazo agudo celular fueron manejados con bolos de metilprednisolona por 3 días y los episodios de rechazo humoral con plasmaféresis, inmunoglobulina y Rituximab según protocolo. 
La inmunosupresión incluyó terapia de inducción con Basiliximab y metilprednisolona al momento de la reperfusión y la de mantención con prednisona, tacrolimus y micofenolato o azatioprina. La profilaxis antibiótica post trasplante en pacientes sin gérmenes aislados previamente fue con tazonam más vancomicina ajustándose según cultivos de donante y receptor. En relación a profilaxis post trasplante hasta el año 2016 en receptores con serología con inmunoglobulina $\mathrm{G}$ positiva para citomegalovirus (IgG $\mathrm{CMV}+$ ) dábamos profilaxis con aciclovir por 4 meses y realizamos seguimiento con carga viral, itraconazol por un año y cotrimoxazol forte trisemanal de por vida. En el año se realizaron modificaciones al protocolo indicando voriconazol por 3 meses y profilaxis universal con valganciclovir por $6 \mathrm{me}-$ ses. El seguimiento post trasplante fue semanal el primer mes, quincenal hasta el tercer mes, mensual hasta el año y luego trimestralmente o según necesidad. Los exámenes realizados en cada control fueron: hemograma, perfil bioquímico, niveles de tacrolimus, espirometría, radiografía de tórax y carga viral para CMV.

Este estudio fue analizado y aprobado por el Comité de Ética de nuestra institución.

\section{Análisis estadístico}

Los datos se presentan mediante promedio y rangos o frecuencias absolutas y relativas según corresponda. Para el análisis de supervivencia se construyeron curvas de Kaplan Meier, censando sujetos vivos al 30 de julio de 2018. Para comparar supervivencia entre grupos se utilizó prueba de log rank, con un nivel de significancia de 0,05 a dos colas. Los análisis se realizaron en el programa Stata 14.0.

\section{Resultados}

Entre agosto de 2010 a julio de 2018 se realizaron 76 trasplantes de los cuales 52 (68\%) fueron por FP. La edad promedio fue 55 años (rango: 43-68), siendo el 53,8\% de género masculino. El IMC promedio pre trasplante fue de 24,4 $\mathrm{kg} / \mathrm{m}^{2}$ (rango: 17-33). En cuanto al diagnóstico fueron 39 FPI (Fibrosis Pulmonar Idiopática), 8 AAE (Alveolitis Alérgica Extrínseca) y 4 casos de IPAF (Interstitial Pneumonia with Autoimmune Features), en un paciente no contamos con resultado histológico del explante. El $61 \%$ de los pacientes tenía criterios ecocardiográficos de Hipertensión Arterial Pulmonar. El LAS promedio fue de 53 y un $32 \%$ cumplía con criterios de urgencia al momento del trasplante (LAS > 45).
En el 98\% de los pacientes el trasplante realizado fue monopulmonar (Tabla 1). Los resultados funcionales (Figura 1) promedio pre trasplante fueron: CVF de $49 \%$ del valor teórico y un test de marcha de $334 \mathrm{~m}$. Los parámetros funcionales a 1 y 3 años post trasplante de CVF fueron 71 y $70 \%$ del valor teórico y un test de marcha 474 y 422 metros respectivamente. La $\mathrm{DL}_{\mathrm{CO}}$ promedio pre trasplante fue de $28,6 \%$ (rango: $15-40 \%$ ). Un $36 \%$ de los pacientes requirió soporte vital extracorpóreo intraoperatorio siendo la hipoxemia persistente, la hipertensión pulmonar severa o la inestabilidad hemodinámica los principales parámetros para indicarla. Desde el año 2010 al año 2014 el soporte vital se realizó con bypass cardiopulmonar convencional y desde el año 2014 el soporte ha sido con ECMO (Extra Corporeal Membrane Oxygenation),

Las principales complicaciones quirúrgicas estuvieron relacionadas a la anastomosis bronquial (8 casos) siendo éstas, estenosis (4 casos), dehiscencia (3 casos) y malacia (1 caso). De las

Tabla 1. Características de 52 pacientes con fibrosis pulmonar trasplantados en el Instituto Nacional del Tórax. 2010-2018

\begin{tabular}{|c|c|}
\hline \multicolumn{2}{|l|}{ Variable } \\
\hline $\begin{array}{l}\text { Género } \mathrm{n}(\%) \\
\text { Femenino } \\
\text { Masculino }\end{array}$ & $\begin{array}{l}25(48 \%) \\
27(52 \%)\end{array}$ \\
\hline Edad $\bar{x}$ (rango) años & $55(43-68)$ \\
\hline $\begin{array}{l}\text { Diagnóstico de base } \\
\text { UIP } \\
\text { AAE } \\
\text { IPAF } \\
\text { No especificado }\end{array}$ & $\begin{array}{c}39(75 \%) \\
8(15,4 \%) \\
4(7,7 \%) \\
1(1,9 \%)\end{array}$ \\
\hline IMC pre trasplante $\overline{\mathrm{X}}$ (rango) $\mathrm{kg} / \mathrm{m}^{2}$ & $24,4(17-33)$ \\
\hline $\begin{array}{l}\text { Tipo de trasplante } \mathrm{n}(\%) \\
\text { Bi pulmonar } \\
\text { Uni pulmonar }\end{array}$ & $\begin{array}{c}1(1,9 \%) \\
51(98,1 \%)\end{array}$ \\
\hline $\begin{array}{l}\text { HTAP } \quad \mathrm{n}(\%) \\
>40 \mathrm{mmHg} \\
\leq 40 \mathrm{mmHg}\end{array}$ & $\begin{array}{l}32(61,5 \%) \\
20(38,5 \%)\end{array}$ \\
\hline $\begin{array}{l}\text { Función pulmonar } \\
\text { CVF }(\overline{\mathrm{X}}, \% \text { valor teórico) } \\
\text { DL }_{\mathrm{CO}}(\overline{\mathrm{x}}, \% \text { valor teórico }) \\
\text { Test de marcha } \overline{\mathrm{x}} \text { (metros) }\end{array}$ & $\begin{array}{l}49 \% \\
28 \% \\
334\end{array}$ \\
\hline Soporte extracopóreo & $19(36 \%)$ \\
\hline
\end{tabular}

*UIP: Neumonía Intersticial Usual; AAE: Alveolitis Alérgica Extrínseca; IPAF: Neumonía Intersticial con Características de Autoinmunidad; IMC: Índice de Masa Corporal; HTAP: Hipertensión Arterial Pulmonar. CVF: Capacidad vital forzada. $\mathrm{DL}_{\mathrm{CO}}$ : Capacidad de difusión de monóxido de carbono; $\overline{\mathrm{X}}=$ promedio. 


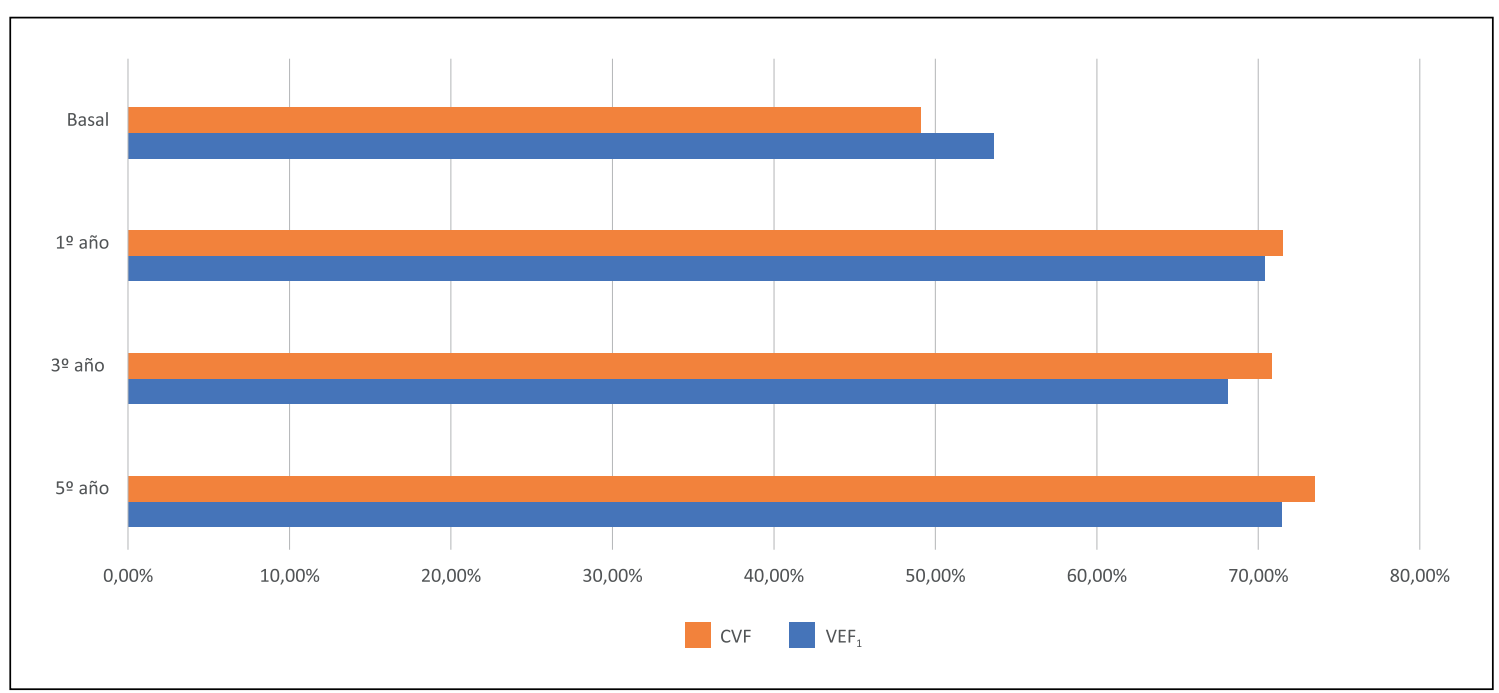

Figura 1. Valores espirométricos en 52 pacientes trasplantados de pulmón por fibrosis pulmonar. Cada par de columnas representa el valor promedio de $\mathrm{CVF}_{\mathrm{y}} \mathrm{VEF}_{1}$ expresados en \% de su valor teórico.

complicaciones médicas precoces destacaron 26 episodios infecciosos y 6 rechazos celulares agudos (Tabla 2). Los cuadros infecciosos bacterianos predominaron, siendo principalmente de origen respiratorio, Pseudomonas y Klebsiella fueron los gérmenes aislados con mayor frecuencia. La infección por Aspergillus se presentó en 7 pacientes siendo 5 bronquitis y dos enfermedades invasivas, un empiema por Aspergillus en el pulmón nativo secundario a neumotórax espontáneo con fuga aérea persistente, manejado en forma exitosa con decorticación pleuropulmonar y un caso que comprometió el injerto pulmonar, ambos tratados con antifúngicos con buena evolución posterior. Cuatro pacientes presentaron infección por citomegalovirus siendo 3 casos de viremia y uno de neumonitis.
En las complicaciones médicas tardías se observó una disminución de los episodios infecciosos registrándose 5 cuadros bacterianos y 6 infecciones virales no CMV. En este período aparecen casos de CLAD en un $32 \%$ de los pacientes, predominando 12 casos de fenotipo obstructivo: 'BOS' y 5 casos de la forma restrictiva: 'RAS'. Cinco pacientes han desarrollado neoplasia: un sarcoma de partes blandas, una leucemia mielocítica aguda tipo M5 y 3 cáncer de pulmón. En estos últimos, en dos casos la biopsia del explante demostró cáncer de pulmón no células pequeñas estadio IA quedando con seguimiento estricto con TAC cada 3 meses, con diagnóstico de recidiva tumoral a los 10 y 14 meses post trasplante respectivamente. El tercer caso de cáncer de pulmón se presentó como una masa en el lóbulo

Tabla 2. Complicaciones médicas precoces y tardías de 52 pacientes trasplantados de pulmón por fibrosis pulmonar. INT 2010-2018

\begin{tabular}{|lcc|}
\hline Complicaciones & Precoces $(<\mathbf{6}$ meses) & Tardías $(>\mathbf{6}$ meses $)$ \\
$\begin{array}{l}\text { Médicas } \\
\text { • Rechazo agudo }\end{array}$ & 6 & $1(\mathrm{RH}) / 8(\mathrm{RCA})$ \\
- Rechazo crónico & $\mathrm{No}$ & $12(\mathrm{BOS}) / 5(\mathrm{RAS})$ \\
Infecciones & $10(38 \%)$ & $5(35,7 \%)$ \\
- Bacterianas & $5(19,2 \%)$ & $6(42,8 \%)$ \\
- Viral no CMV & $4(15,3 \%)$ & - \\
- Viral CMV & $7(26,9 \%)$ & $3(21,4 \%)$ \\
- Hongos & No & 5 \\
\hline Neoplásicas & & 5 \\
\hline
\end{tabular}

$\mathrm{CMV}=$ citomegalovirus; $\mathrm{RH}=$ rechazo humoral; $\mathrm{RCA}=$ rechazo celular agudo; $\mathrm{BOS}=$ síndrome de bronquiolitis obliterante; RAS = síndrome del injerto restrictivo. 


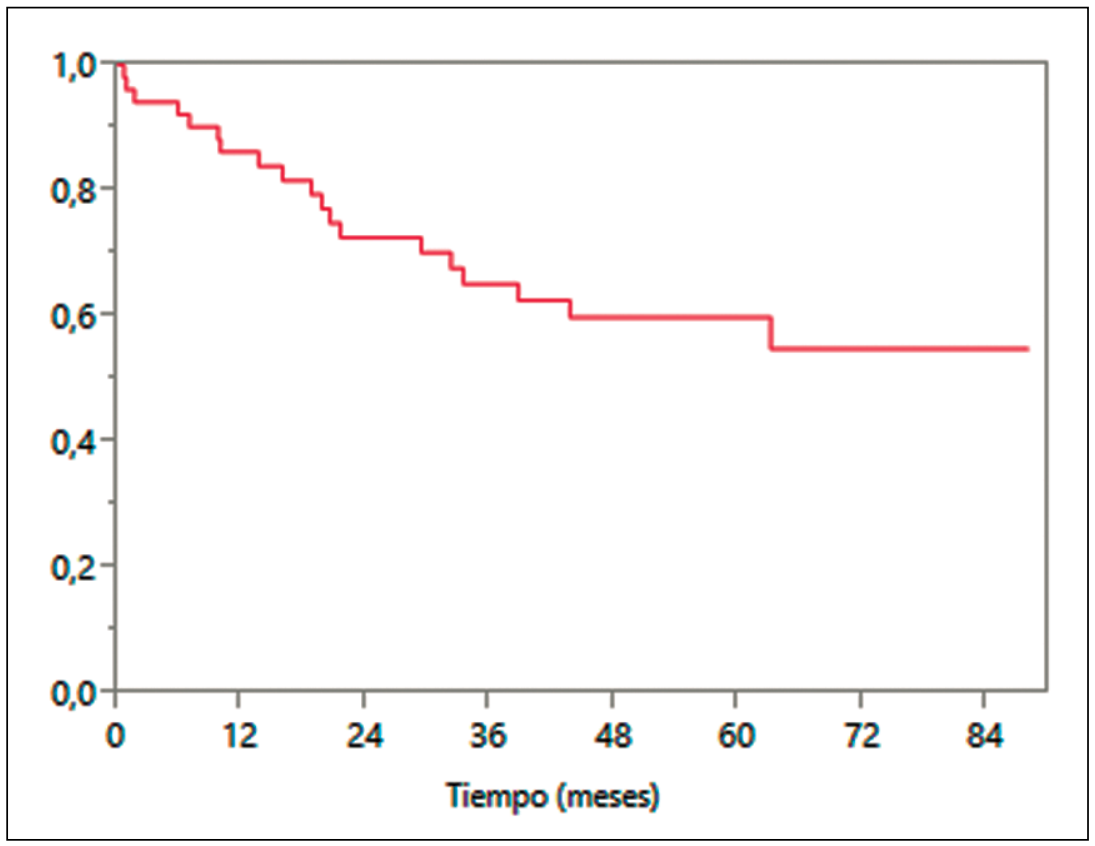

Figura 2. Curva de sobrevida de 52 pacientes trasplantados de pulmón por fibrosis pulmonar (Kaplan Meier). INT 2010-2018.

inferior izquierdo del pulmón nativo con biopsia positiva para cáncer escamoso (Tabla 2). Complicaciones de menor frecuencia fueron 2 pacientes con SAHOS (Síndrome de Apnea-Hipopnea del Sueño), 1 episodio de pancreatitis por fármacos y 2 con RGE (Reflujo Gastro-Esofágico) operados que requirieron cirugía antirreflujo.

En el seguimiento 21 pacientes han fallecido $(40 \%)$, siendo las principales causas infecciosas (42\%), CLAD (28\%) y por neoplasias (19\%). Tuvimos 5 fallecidos en los primeros 6 meses, 4 por sepsis respiratoria de origen bacteriano y un caso por arritmia ventricular. Después de los 6 meses 16 fallecieron: 6 casos por CLAD, 5 de origen infeccioso (3 de origen bacteriano y 2 virales), 4 por neoplasias y un caso de pancreatitis asociada a fármacos.

La sobrevida de esta cohorte a 1, 3 y 5 años fue de 86,2, 65,2 y 59\% respectivamente (Figura 2 ). Se comparó curva de supervivencia de pacientes con FPI, AAE e IPAF no encontrándose diferencias significativas en los grupos.

\section{Discusión}

La principal conclusión de este trabajo es que el trasplante monopulmonar es una opción viable y segura de tratamiento en nuestro medio para pacientes con enfermedades pulmonares intersticiales en etapa terminal con buenos resultados en sobrevida comparables a datos internacionales.
En el último reporte de la ISHLT la sobrevida de pacientes con FP a 1, 3 y 5 años fue de $78 \%, 65 \%$ y $50 \%$ respectivamente, siendo resultados globales que incluyen a trasplantes mono y bipulmonares ${ }^{2}$. Existe una tendencia mundial a un aumento progresivo en la realización de trasplantes bipulmonares independiente del diagnóstico y datos recientes muestran ventajas en sobrevida en estos pacientes con $\mathrm{FP}^{2}$. Nuestro programa ha privilegiado el trasplante monopulmonar $(98 \%$ en nuestra serie) por diversas razones, tales como: menor tiempo en lista de espera, tiempos quirúrgicos más cortos, menos complicaciones perioperatorias y la escasez de órganos que determinan que un donante potencial pueda beneficiar a dos pacientes al mismo tiempo. La sobrevida en nuestra serie a 1,3 y 5 años fue de $86 \%, 65 \%$ y $59 \%$ respectivamente, resultados ligeramente mejores que la sobrevida de trasplantes monopulmonares del registro internacional que a 1,3 y 5 años fue de 77,59 y $45 \%$ respectivamente ${ }^{2}$. Sin embargo, en un equipo con experiencia creciente y con buenos resultados, la tendencia también será a preferir el trasplante bipulmonar cuando sea posible, evitando complicaciones de los monopulmonares y optando a una mejor sobrevida.

Cuando se analizan los factores de riesgo de mayor mortalidad a 1 y 5 años se debe considerar: la edad del donante y receptor, IMC y el volumen de trasplantes por centro. Datos del registro muestran que centros que realizan $5 \mathrm{TP}$ en el año tienen un riesgo de mortalidad al año de 
un 50\% mayor, comparado con aquellos centros que trasplantan 38 pacientes por año (HR1, IC 95\%). En una publicación reciente de Thabut et $\mathrm{al}^{7}$, se muestra la sobrevida de centros de TP en Estados Unidos encontrando que aquellos que realizan menos de $10 \mathrm{TP}$ por año tienen tasas de sobrevida de $30 \%$ a 5 años. Estos reportes no se condicen con nuestros buenos resultados, donde realizamos en promedio 9 TP por año situándonos en el grupo de centros de bajo volumen.

Los resultados favorables probablemente están explicados en parte por el control de los factores de riesgo que se asocian a resultados adversos post trasplante. En nuestro programa la edad máxima de ingreso a lista son 65 años, uso muy limitado de donantes marginales, una intervención y monitoreo nutricional estricto considerando contraindicado el TP en pacientes con pesos extremos y, la implementación de un programa de ejercicio y rehabilitación pre y post trasplante que es mandatorio en todo paciente que ingresa a lista de espera. Todo lo anterior se complementa con un equipo multidisciplinario, insertos en un hospital de alta complejidad con experiencia en cirugía torácica y cardíaca.

Después del post operatorio inmediato la principal complicación que se asocia a la inmunosupresión y a la exposición del pulmón implantado con el exterior, son las infecciones. Es la causa de un 35\% de las muertes dentro de los primeros 6 meses. El tipo de infección depende del tiempo que se presente después del trasplante, predominando las de origen nosocomial los primeros meses, luego aumentan infecciones oportunistas y reactivación de infecciones latentes, después del primer año a medida que el paciente se reinserta en la sociedad aumenta el riesgo de infecciones adquiridas en la comunidad ${ }^{8}$. En nuestra serie las principales complicaciones infecciosas se observaron dentro de los primeros 6 meses (26 episodios) coincidiendo con el período de máxima inmunosupresión, siendo de origen bacteriano la gran mayoría, luego estas van disminuyendo con el tiempo. La infección fue la principal causa de mortalidad, en efecto, un $42 \%$ (9 casos) de los pacientes falleció por infección, 4 de ellos en los primeros 6 meses. Las infecciones fúngicas son una causa importante de morbimortalidad; en nuestra serie un $27 \%$ de las infecciones en los primeros 6 meses fueron por esta causa. La mayor prevalencia es probablemente debido a varios factores entre ellos: trasplante monopulmonar con pulmón nativo con lesiones quísticas susceptibles de ser colonizadas y a factores ambientales como la gran cantidad de construcciones en la Región Metropolitana. Se presentaron como bronquitis, micetomas en pulmón nativo y dos casos de enfermedad invasiva por Aspergillus sp. Debido a estos cuadros, cambiamos la profilaxis de itraconazol por voriconazol en todos los pacientes y se optará por la técnica bipulmonar en los pacientes con daño estructural severo por FP.

Después del primer año las principales complicaciones son CLAD y las neoplasias. El CLAD, es el principal limitante del trasplante a largo plazo con una incidencia de $12 \%$ al año y $50 \%$ a 5 años. Hay dos subtipos, uno obstructivo y más frecuente llamado BOS $(70 \%)$ y uno restrictivo, RAS (30\%), caracterizado por lesiones fibróticas periféricas de predominio superiores, de peor pronóstico que el $\mathrm{BOS}^{9}$. En nuestra serie un 32\% de los pacientes ha cursado con CLAD siendo un $70 \%$ de tipo BOS similar a los datos internacionales. La disfunción crónica del injerto ha sido la causa de muerte del $28 \%$ de los pacientes. Hasta el momento no hay terapias efectivas para el manejo de esta complicación.

La presencia de neoplasias fue otra complicación tardía que es importante analizar. Se estima que tienen una incidencia de $13 \%$ a los 5 años y $28 \%$ a los 10 años. Los más frecuentes son los cánceres de piel no melanoma, neoplasias de pulmón nativo en 7,9\% y desórdenes linfoproliferativos en 2 a $8 \%$. Entre los factores de riesgo que se describen para desarrollar neoplasia son el tipo y tiempo de inmunosupresión, el sexo masculino, edad $>55$ años, la historia de tabaquismo, el trasplante monopulmonar y una nueva adquisición del virus Epstein-Barr ${ }^{10}$. En nuestra serie, cinco pacientes han desarrollado neoplasias: un sarcoma de partes blandas, una leucemia mielocítica aguda tipo M5 y 3 neoplasias de pulmón. En estos últimos, en dos casos la biopsia del explante demostró cáncer de pulmón no células pequeñas estadio IA quedando con seguimiento estricto con TAC cada 3 meses, en ambos hubo recidiva local a los 10 y 14 meses post trasplante. Dado el mayor riesgo de neoplasias pulmonares se realiza TAC de tórax de control cada 6 meses y PET/CT en caso de lesiones nodulares sospechosas.

Desde el punto de vista funcional, todos fueron dados de alta sin requerimientos de oxígeno suplementario y mejoraron progresivamente los resultados funcionales reflejados en la CVF y en el test de caminata de 6 min.

\section{Conclusión}

En pacientes con fibrosis pulmonar en fase avanzada el trasplante monopulmonar es una opción segura con buenos resultados en sobrevida. 
Una derivación precoz, una cuidadosa selección y preparación del candidato a trasplante, junto al trabajo de un equipo multidisciplinario son determinantes en el exitoso desarrollo de un programa de trasplante de bajo volumen como el nuestro.

Las infecciones son la principal causa de mortalidad en receptores de trasplante pulmonar, el adecuado estudio pre trasplante, la estrategias de profilaxis en el momento del trasplante y el tratamiento oportuno pueden mejorar los resultados.

La disfunción crónica de injerto es la principal limitante de la sobrevida a largo plazo y hasta el momento no existen terapias efectivas para enfrentarla.

\section{Bibliografía}

1.- WEILL D, BENDEN C, CORRIS P, DARK J, DAVIS $\mathrm{R}, \mathrm{KESHAVJEE} \mathrm{S}$, et al. A consensus document for the selection of lung transplant candidates: 2014-An update from the Pulmonary Transplantation Council of the International Society for Heart and Lung Transplantation. J Heart Lung Transplant 2015; 34: 1-15.

2.- CHAMBER D, CHERIKH W, GOLDFARB S, HAYES D, KUCHERYAVAYA A, TOLL A, et al International Thoracic Organ Transplant Registry of the International Society for Heart and Lung Transplantation: Thirty-first adult lung and heart-lung transplantation report 2018. J Heart Lung Transplant 2018; 37: 1169-83.

3.- TRAVIS W, COSTABEL U, HANSELL D, KING T,
LYNCH D, NICHOLSON A, et al. An Official American Thoracic Society/European Respiratory Society Statement: Update of the International Multidisciplinary Classification of the Idiopathic Interstitial Pneumonias. Am J Respir Crit Care Med 2013, 188: 733-48.

4.- MERLO C, WEISS E, ORENS J, BORJA M, WEST M, CONTE J. Impact of U.S Lung Score on Survival After Lung Transplantation. J Heart Lung Transplant 2009; 28: 769-75.

5.- HOVANESSIAN A, WALLACE W, LYNCH J, BELPERIO J. Chronic Lung Allograft Dysfunction: Envolving Concepts and Therapies. Semin Resp Crit Care Med 2018; 39: 155-71.

6.- LEVINE D, GLANVILLE A, ABOYOUN C, BELPERIO J, BENDEN C, BERRY G, et al. Antibodymediated rejection of the lung: A consensus of the International Society for Heart and Lung Transplantation. J Heart Lung Transplant 2016; 35: 397-406.

7.- THABUT G, CHRISTIE JD, KREMERS WK, FOURNIER M, HALPEM SD. Survival differences following lung transplantation among US transplant centers. JAMA 2010; 304: 53-60.

8.- HERNÁNDEZ R, AGUILAR M, CARRASCO M, USETTI P. Lung transplantation idiophatic pulmonary fibrosis. Med I 2018; 6: 68.

9.- KUMAR A, KAPNADAK S, GIRGIS R, RAGHU G. Lung transplantation in idiophatic pulmonary fibrosis. Expert Rev Respir Med 2018; 12: 375-85.

10.- OLLAND A, FALCOZ P, MASSARD G. Malignancies after lung transplantation. J Thorac Dis 2018; 10: 3132 40.

Correspondencia a:

Dr. Joel Melo Tanner

Instituto Nacional del Tórax

José M. Infante 717. Providencia.

Santiago, Chile.

Email: joelmelot@hotmail.com 